\title{
Enhancement of ketorolac tromethamine permeability through rat skin using penetration enhancers: An ex-vivo study
}

\author{
Pawan Kumar, Shailendra Kumar Singh, Dina Nath Mishra, Priti Girotra \\ Department of Pharmaceutical Sciences, Guru Jambheshwar University of Science \& Technology, Hisar, Haryana, India
}

\begin{abstract}
Introduction: Ketorolac tromethamine (KT), a nonsteroidal anti-inflammatory drug, when given orally causes gastrointestinal disturbances. Its transdermal drug delivery may reduce such side effects associated with them. The present investigation was aimed at evaluating the efficiency of various penetration enhancers for improved permeation of $\mathrm{KT}$ through the skin. Materials and Methods: A concentration of $1 \mathrm{mg} / \mathrm{mL}$ of the drug solution with enhancers was used to evaluate diffusion through the rat skin using a Franz diffusion cell assembly. 20 different penetration enhancers were selected for this study. Results: Saturated fatty acids like stearic and palmitic acid were found to increase the permeation rate of the drug to a great extent whereas unsaturated fatty acid viz. oleic acid exhibited maximum permeation. Increase in permeability efficiency of various penetration enhancers was observed in the following order: Oleic acid $>$ stearic acid $>$ palmitic acid $>$ isopropyl myristate $>$ tween $80>$ span $80>$ span $40>$ span $20>$ I-limonene $>$ I-menthol $>$ fenchone $>\alpha$-pinene $>$ urea $>$ dimethyl sulfoxide $($ DMSO) $>$ triton $X-100>$ tween $20>$ dimethyl formamide $>$ acetone $>$ control $>$ citric acid $>$ ascorbic acid. Ascorbic acid and citric acid had no effect on permeation rate. Conclusion: The results revealed that the permeation of KT through the skin can maximally be enhanced using oleic acid-an unsaturated fatty acid.
\end{abstract}

Key words: Ketorolac tromethamine, penetration enhancer, skin permeation, transdermal drug delivery system

\section{INTRODUCTION}

Transdermal drug delivery system, a topic of increasing interest to researchers, is the delivery of a therapeutic agent through the intact skin for the exertion of its systemic effect. The drugs having short biological half-lives can be administered through this route as they can avoid first pass metabolism. ${ }^{[1,2]}$ For the effective permeation of drugs through the skin, various penetration enhancers are incorporated in the formulation along with the therapeutic agent for improving the permeation through the skin. Some of the penetration enhancers possess the potential to cause

\section{Address for correspondence:}

Pawan Kumar,

Department of Pharmaceutical Sciences, Guru Jambheshwar

University of Science \& Technology, Hisar - 125001 , Haryana, India.

E-mail: pawansoni528@gmail.com

\begin{tabular}{|l|l|}
\hline \multicolumn{2}{|c|}{ Access this article online } \\
\hline Quick Response Code: & Website: \\
\hline & www.jpionline.org \\
\cline { 2 - 2 } & DOI: \\
\hline
\end{tabular}

skin irritation. Hence, preferably those enhancers which have been listed under generally recognized as safe agents by U.S.A food and drug administration (e.g., terpenes and essential oils) must be used for transdermal applications. ${ }^{[3]}$

Ketorolac tromethamine (KT), a pyrrolizine carboxylic acid derivative, is being used as a potent nonnarcotic analgesic in the treatment of moderate to severe postoperative pain. ${ }^{[4]}$ It has been included in the treatment regimen of pain associated with abdominal, gynecologic, orthopedic and urologic surgery. ${ }^{[5]}$

Ketorolac tromethamine demonstrates an oral bioavailability of $90 \%$ with a very low first pass metabolism. However, upon oral administration, it exhibits adverse effects such as gastrointestinal ulceration and has a short biological half-life (4-6 h). In order to avert these adverse effects, a transdermal drug delivery system has been explored. ${ }^{[6]}$

In the present investigation, 20 penetration enhancers belonging to ten different chemical classes were selected and analyzed for the permeation enhancement of the drug KT using ex-vivo diffusion study through the rat skin.

Large number of penetration enhancers were judiciously selected for the present study to represent diverse chemical structures 
(such as chain length, polarity, level of unsaturation and presence of some groups such as alcohols, sulfur, ketones, esters, amide), as the interaction between enhancers and stratum corneum (SC) may differ resulting in variable efficiency as permeation enhancers.

\section{MATERIALS AND METHODS}

\section{Materials}

Ketorolac tromethamine was generously gifted by Ranbaxy Laboratories Ltd. (Gurgaon, India). a-pinene, l-limonene, and fenchone were purchased from Sigma Chemical Company (St. Louis, USA). Propylene glycol (PG), acetone, dimethyl sulfoxide, dimethyl formamide, ascorbic acid, citric acid, isopropyl myristate, tweens $(20,80)$ and spans (20, 40,80 ), and triton X-100 were obtained from Hi-Media, Mumbai, India. All other chemicals utilized were of suitable analytical grade.

\section{Animals}

Wistar rats (200-250 g) were procured from the disease-free small animal house, Lala Lajpat Rai University of Veterinary and Animal Sciences, Hisar, Haryana. The experimental protocol was approved by the Institutional Animals Ethics Committee (registration number 0436).

\section{Methods}

\section{Preparation of rat skin}

Abdominal skin of Wistar rats (200-250 g) was used for the permeation studies. The day before harvesting of the skin, the hair on the dorsal side of the animal was removed using marketed depilator $\left(\right.$ Veet $\left.^{\circledR}\right)$ taking extreme precaution not to damage the skin. The rats were sacrificed by cervical dislocation and the hairless skin was excised from the animal. It's subcutaneous fat was surgically removed, and the skin pieces were wiped with $0.1 \% \mathrm{w} / \mathrm{v}$ trypsin solution for $2 \mathrm{~h} .{ }^{[7]}$

\section{Skin permeation studies}

Franz-type diffusion cell having a diffusional surface area of $1.12 \mathrm{~cm}^{2}$ was used for permeation studies. Phosphate buffer ( $\mathrm{pH} 7.4)$ was used as receptor fluid. Franz diffusion cells were then filled with receptor fluid and equilibrated at $37^{\circ} \mathrm{C}$ for $1 \mathrm{~h}$. Freshly excised full-thickness rat skin was mounted between donor and receptor compartments. SC was arranged to face toward the donor compartment. Test solution $(2.0 \mathrm{~mL})$ was applied to the SC side of the donor compartment. Throughout the experiment, the receptor chamber content was continuously agitated by a magnetic stirrer at $37 \pm 0.5^{\circ} \mathrm{C}$. Aliquots $(2.0 \mathrm{~mL})$ were withdrawn from the receptor compartment at an interval of $0.5,1,2,3,4$, and $6 \mathrm{~h}$ and analyzed for drug content. The sample volume was replenished with the fresh receptor medium as and when the samples were withdrawn. The drug content was determined by measuring the absorbance at $323 \mathrm{~nm}$ using ultraviolet-visible double beam spectrophotometer (modelsystronics 2203).

\section{Preparation of vehicles}

The vehicles were prepared by dissolving appropriate amounts of the drug and the enhancers directly in PG. PG was selected so that both hydrophilic and hydrophobic adjuvant could be dissolved. The arbitrary concentration of enhancers was $10 \%$ (w/v or v/v). A control PG vehicle containing $1 \% \mathrm{KT}$ and no penetration enhancer was used as a reference.

\section{Calculation of permeability coefficient}

To calculate the permeation parameters, a graph of penetrated amount of the drug versus time was plotted. The steady state flux (Jss) was calculated from the slope of the graph.

The cumulative drug permeation $(Q t)$ was calculated using the following equation:

$Q t=V r C t+\sum_{i=0}^{t-1} V_{s} C i$

Where $C t$ is the drug concentration of the receiver solution at each sampling time, $C i$ is the drug concentration of the $i$ th sample, and $V \mathrm{r}$ and $V \mathrm{~s}$ are the volumes of the receiver solution and the sample, respectively.

Data were expressed as the cumulative $Q t /$ unit of skin surface area, $Q t / S\left(S=1.12 \mathrm{~cm}^{2}\right)$. The $J$ ss were calculated by linear regression interpolation of the experimental data at a steady state:

$\mathrm{Jss}=\frac{\Delta Q t}{(\Delta t \times S)}$

Apparent permeability coefficients $(K \mathrm{p})$ were calculated according to the equation:

$\mathrm{Kp}=\frac{\mathrm{Jss}}{C d}$

Where $C d$ is the drug concentration in the donor compartment.

The enhancement ratio (ER) was calculated from following equation. ${ }^{[8]}$

$\mathrm{ER}=\frac{\mathrm{Kp} \text { with enhancer }}{\mathrm{Kp} \text { without enhancer }}$

\section{Statistical analysis}

The statistical analysis was performed using the software GraphPad Instat (DataSet3.ISD). The results were analyzed using one-way ANOVA and then by Dunnett's $t$-test. $P<0.001$ was considered as statistically significant.

\section{RESULTS AND DISCUSSION}

The flux (J), permeability coefficient (Kp), and ER for each of the different enhancers obtained through studies of drug solution with concentration $(1 \mathrm{mg} / \mathrm{mL})$ through rat skin and calculated according to equations (1), (2), (3), and (4) have been tabulated in Table 1 . 


\begin{tabular}{|c|c|c|c|}
\hline $\begin{array}{l}\text { Penetration } \\
\text { enhancers }\end{array}$ & $\mathrm{J}\left(\mu \mathrm{gcm}^{-2} \mathrm{~h}^{-1}\right)$ & $\mathrm{Kp}\left(\mathrm{cm} \cdot \mathrm{h}^{-1}\right)$ & ER \\
\hline Control & $71.47 \pm 0.625$ & $0.0350 \pm 0.00035$ & 1 \\
\hline$\alpha$-Pinene & $199.70 \pm 2.23$ & $0.1997 \pm 0.00223$ & 5.70 \\
\hline I-Menthol & $212.63 \pm 3.80$ & $0.2126 \pm 0.00380$ & 6.07 \\
\hline Span 80 & $379.93 \pm 5.61$ & $0.3799 \pm 0.00561$ & 10.85 \\
\hline Span 40 & $305.13 \pm 3.32$ & $0.3051 \pm 0.00332$ & 8.71 \\
\hline Stearic acid & $507.73 \pm 2.15$ & $0.5077 \pm 0.00215$ & 14.50 \\
\hline Isopropyl myristate & $440.93 \pm 5.30$ & $0.4409 \pm 0.00530$ & 12.59 \\
\hline DMSO & $153.03 \pm 1.55$ & $0.1530 \pm 0.00155$ & 4.37 \\
\hline DMF & $99.63 \pm 1.26$ & $0.0996 \pm 0.00126$ & 2.84 \\
\hline Triton X-100 & $152.23 \pm 2.81$ & $0.1522 \pm 0.00281$ & 4.34 \\
\hline Citric acid & $17.92 \pm 1.09$ & $0.0179 \pm 0.00109$ & 0.51 \\
\hline Span 20 & $236.66 \pm 6.31$ & $0.2366 \pm 0.00631$ & 6.76 \\
\hline Fenchone & $206.93 \pm 6.43$ & $0.2069 \pm 0.00643$ & 5.91 \\
\hline Oleic acid & $514.43 \pm 2.50$ & $0.5144 \pm 0.00250$ & 14.69 \\
\hline Ascorbic acid & $12.50 \pm 0.79$ & $0.0125 \pm 0.00079$ & 0.35 \\
\hline Palmitic acid & $474.33 \pm 1.15$ & $0.4743 \pm 0.00115$ & 13.55 \\
\hline Tween 80 & $411.30 \pm 1.50$ & $0.4113 \pm 0.00150$ & 11.75 \\
\hline Urea & $154.06 \pm 2.50$ & $0.1540 \pm 0.002 .50$ & 4.40 \\
\hline I-limonene & $235.99 \pm 3.19$ & $0.2359 \pm 0.00319$ & 6.73 \\
\hline Acetone & $75.59 \pm 1.72$ & $0.0755 \pm 0.00172$ & 2.15 \\
\hline Tween 20 & $146.23 \pm 0.94$ & $0.1462 \pm 0.00094$ & 4.17 \\
\hline
\end{tabular}

The permeation rate of the KT showed enhanced permeation with fatty acids. Among the saturated fatty acids, with stearic acid, the permeability flux $(\mathrm{J})$ was $507.73 \pm 2.15\left(\mu \mathrm{gcm}^{-2} / \mathrm{h}\right)$ which was 14.50-fold more than that of KT without a permeation enhancer $\left(71.47 \pm 0.625 \mu \mathrm{gcm}^{-2} / \mathrm{h}\right)$. The addition of the unsaturated fatty acids, oleic acid (C18:1) exhibited the maximum permeation rate $\left(514.43 \pm 2.50 \mu \mathrm{gcm}^{-2} / \mathrm{h}\right)$ as compared to the presence of the corresponding saturated fatty acid. Unsaturated fatty acid like oleic acid (C18:1) exhibited maximum permeation $\left(\mathrm{J}=514.43 \pm 2.50 \mu \mathrm{gcm}^{-2} / \mathrm{h}\right)$.

On the other hand, terpenes (l-1imonene, $\alpha$-pinene, 1 -menthol, and fenchone) resulted in a 5 to 7 -fold increase in permeability coefficient.

In the case of nonionic surfactants that is, tweens $(20,80)$ and spans $(20,40,80)$, tween 80 demonstrated the highest permeation rate. The structure of tweens and spans is relevant to this role. The structure imparts both lipophilic and hydrophilic characteristics to the enhancer, allowing it to partition between lipophilic mortar substance and the hydrophilic protein domains. Nonionic surfactant increases rate of transport by two possible mechanisms, one by penetration into the intercellular regions of SC by solubilizing lipid component, thereby increasing the fluidity, secondly disruption of comeocyte by interaction and binding with keratin filament. ${ }^{[9,10]}$

The flux of KT was significantly higher in the presence of DMSO, triton X-100, and isopropyl myristate (IPM), when compared with the control. The effect of DMSO on skin permeation can be explained as the consequence of denaturation of proteins and perturbation of the bilayer lipid layer packing in the keratinized SC layer. ${ }^{[11]}$

Acetone and DMF also increased the permeation rate, but ascorbic acid and citric acid did not show any increase in permeability of drug. The aprotic penetration enhancer, DMF, probably forms solvation shell formation surrounding the polar head groups of the lipid and hence increases the permeation. ${ }^{[12]}$

Figure 1 indicates the permeation profile of all the penetration enhancers used in the present study, wherein, it can be observed that oleic acid exhibits the maximum permeation rate.

Isopropyl myristate was able to show a high enhancing effect, due to very effective penetration through nonpolar route in the skin where they disrupt lipid layer and increase fluidity. ${ }^{[13]}$

Urea increases the permeability coefficient of KT by altering the barrier function of the skin through the increase of the hydration of the SC and inducement of keratolysis of skin after prolonged contact. $^{[14]}$

Fatty acids are the most attractive skin permeation enhancers having lipophilic properties; many studies have shown that the skin permeability enhancing property increases with PG vehicles. ${ }^{[15]}$ The solubility of KT in PG remained unaffected by adding the fatty acid. The increase in the permeation rate of KT with fatty acids plausibly resulted in the increased permeation coefficient. The double bonds present in unsaturated fatty acids, like oleic acid, alter the lipid structure of the skin by producing "kink," isomer, which facilitates the permeation of drug across the skin. ${ }^{[16,17]}$

The enhancing activity of terpenes, which presumably act by reversibly modifying the barrier properties of the SC and by increasing the drug partitioning into the skin barrier, has been reported in the case of several model penetrants, such as estradiol, ${ }^{[18]} 5$-fluorouracil, ${ }^{[19]}$ and indomethacin. ${ }^{[20]}$

\section{CONCLUSION}

It can thus be concluded that out of the 20 penetration enhancers, studied for the flux, permeability coefficient and ER, unsaturated fatty acid (oleic acid) increased the permeation rate of KT more than the saturated fatty acids (stearic and palmitic acid). Terpenes and nonionic surfactants also increased the permeation by penetrating into the intercellular regions of SC. DMSO, triton X-100, and IPM increased the ER to an extent of 4-5 folds. Acetone, urea, and DMF also increased the permeability of KT by increasing the hydration of the SC and then causing keratolysis of skin. Ascorbic acid and citric acid did not increase the 


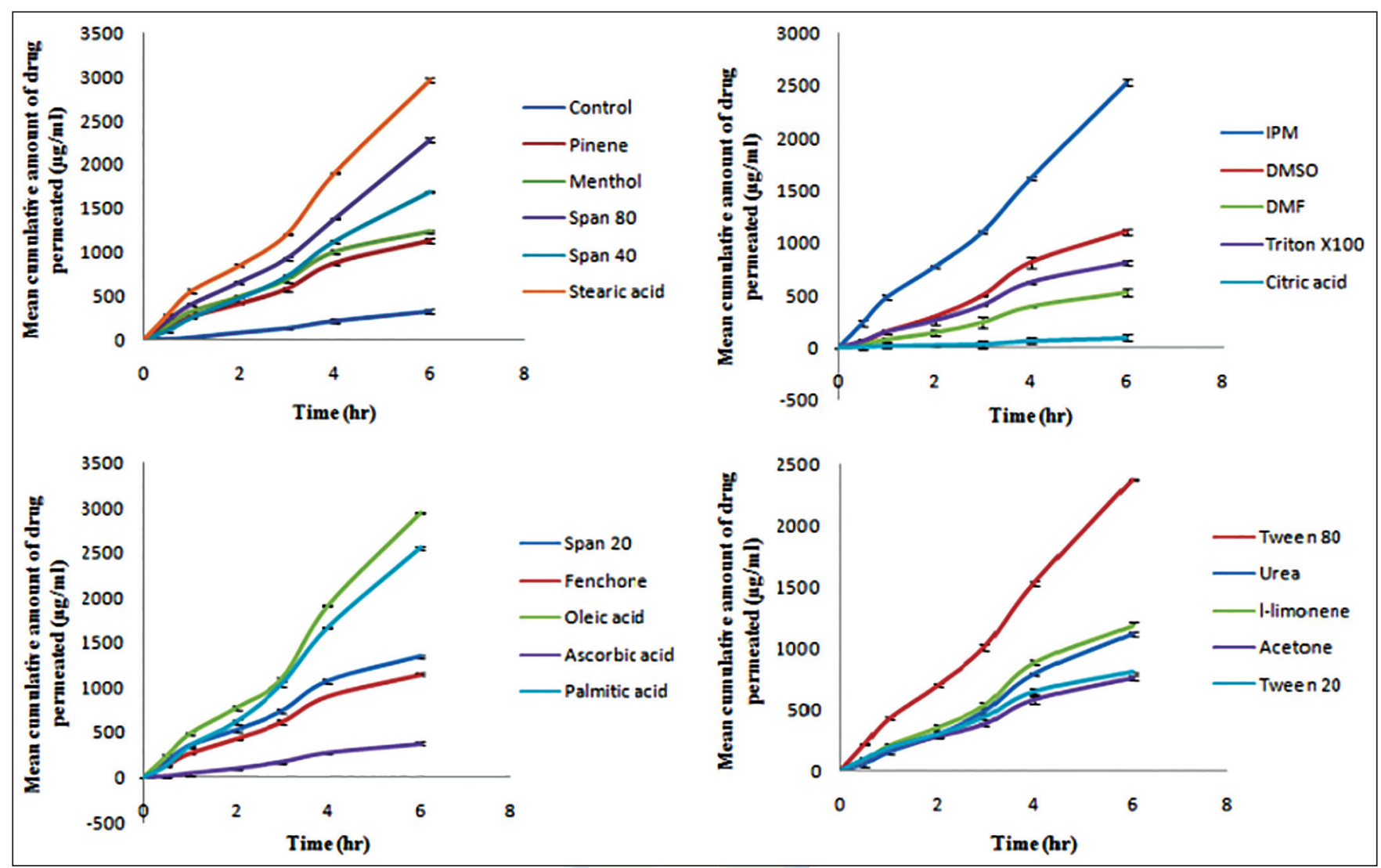

Figure 1: Mean cumulative amount of ketorolac tromethamine permeated through $1.12 \mathrm{~cm}^{2}$ of rat skin for $6 \mathrm{~h}$ in the presence of penetration enhancers from the saturated drug solution. Each point represents mean \pm standard deviation $(n=3)$

permeation rate of the drug. Thus, the present study may provide a platform for researchers to optimize the penetration enhancement of different penetration enhancers for the transdermal delivery of KT.

\section{ACKNOWLEDGEMENT}

The authors extend their sincere thanks to All India Council of Technical Education, New Delhi for financial support. The authors wish to thank Dr. Sangita Saini for her help and guidance in relation to this manuscript.

\section{REFERENCES}

1. Tiwari SB, Udupa $\mathrm{N}$. Investigation into the potential of iontophoresis facilitated delivery of ketorolac. Int J Pharm 2003;260:93-103.

2. Roy SD, Manoukian E, Combs D. Absorption of transdermally delivered ketorolac acid in humans. J Pharm Sci 1995;84:49-52.

3. Sapra B, Jain S, Tiwary AK. Percutaneous permeation enhancement by terpenes: Mechanistic view. AAPS J 2008;10:120-32.

4. Rooks WH, Maloney PJ, Shott LD, Schuler ME, Sevelius H, Strosberg AM, et al. The analgesic and anti-inflammatory profile of ketorolac and its tromethamine salt. Drugs Exp Clin Res 1985;11:479-92.

5. Sinha VR, Kumar RV, Singh G. Ketorolac tromethamine formulations: An overview. Expert Opin Drug Deliv 2009;6:961-75.
6. Buckley MM, Brogden RN. Ketorolac. A review of its pharmacodynamic and pharmacokinetic properties, and therapeutic potential. Drugs 1990;39:86-109.

7. Panchagnula R, Salve PS, Thomas NS, Jain AK, Ramarao P. Transdermal delivery of naloxone: Effect of water, propylene glycol, ethanol and their binary combinations on permeation through rat skin. Int $\mathrm{J}$ Pharm 2001;219:95-105.

8. Williams AC, Barry BW. Essential oils as novel human skin penetration enhancers. Int J Pharm 1989;57:R7-9.

9. Breuer MM. The interaction between surfactants and keratinous tissues. J Soc Cosmet 1979;30:41-64.

10. Walters KA, Walker M, Olejnik O. Non-ionic surfactant effects on hairless mouse skin permeability characteristics. J Pharm Pharmacol 1988;40:525-9.

11. Williams AC, Barry BW. Penetration enhancers. Adv Drug Deliv Rev 2004;56:603-18.

12. Walker RB, Smith EW. The role of percutaneous penetration enhancers. Adv Drug Deliv Rev 1996;18:295-301.

13. Panigrahi L, Pattnaik $\mathrm{S}$, Ghosal SK. The effect of $\mathrm{pH}$ and organic ester penetration enhancers on skin permeation kinetics of terbutaline sulfate from pseudolatex-type transdermal delivery systems through mouse and human cadaver skins. AAPS PharmSciTech 2005;6:E167-73.

14. Hardgaft J. Penetration enhancers in percutaneous absorption. Pharm Int 1984;5:252-4.

15. Cooper ER. Increased skin permeability for lipophilic molecules. J Pharm Sci 1984;73:1153-6.

16. Aungst BJ, Rogers NJ, Shefter E. Enhancement of naloxone penetration through human skin in vitro using fatty acids, fatty 
alcohols, surfactants, sulphoxides and amides. Int $\mathrm{J}$ Pharm 1986;33:225-34.

17. Kandimalla K, Kanikkannan N, Andega S, Singh M. Effect of fatty acids on the permeation of melatonin across rat and pig skin in-vitro and on the transepidermal water loss in rats in-vivo. J Pharm Pharmacol 1999;51:783-90.

18. Williams AC, Barry BW. The enhancement index applied to terpene penetration enhancers for human skin and model lipophilic (oestradiol) and hydrophilic (5-fluorouracil) drugs. Int J Pharm 1991;74:157-68.

19. Williams AC, Barry BW. Human Skin Penetration Enhancement: The Synergy of Propylene Glycol with Terpenes. In Proceedings of $16^{\text {th }}$ International Symposium on
Controlled Release on Bioactive Materials. Vol. 16. Chicago, USA; 1989. p. 33-4.

20. Okabe H, Takayama K, Ogura A, Nagai T. Effect of limonene and related compounds on the percutaneous absorption of indomethacin. Drug Des Deliv 1989;4:313-21.

How to cite this article: Kumar P, Singh SK, Mishra DN, Girotra P. Enhancement of ketorolac tromethamine permeability through rat skin using penetration enhancers: An ex-vivo study. Int J Pharma Investig 2015;5:142-6.

Source of Support: The present work was financially supported by All India Council of Technical Education, New Delhi. Conflicts of Interest: The authors alone are responsible for the content and writing of the paper. 